\title{
A microfluidic device for simple and rapid evaluation of multidrug efflux pump inhibitors
}

\author{
Ryota lino ${ }^{1,2}$, Kunihiko Nishino ${ }^{3}$, Hiroyuki Noji ${ }^{1,2}$, Akihito Yamaguchi ${ }^{4,5}$ and Yoshimi Matsumoto * \\ ' Department of Applied Chemistry, Graduate School of Engineering, University of Tokyo, Bunkyo-ku, Tokyo, Japan \\ ${ }^{2}$ Core Research for Evolutional Science and Technology, Japan Science and Technology Agency, Chiyodaku, Tokyo, Japan \\ ${ }^{3}$ Laboratory of Microbiology and Infectious Diseases, Institute of Scientific and Industrial Research, Osaka University, Ibaraki, Osaka, Japan \\ ${ }^{4}$ Department of Cell Membrane Biology, Institute of Scientific and Industrial Research, Osaka University, Ibaraki, Osaka, Japan \\ ${ }^{5}$ Graduate School of Pharmaceutical Sciences, Osaka University, Suita, Osaka, Japan
}

\section{Edited by:}

Tzi Bun Ng, The Chinese University of Hong Kong, China

\section{Reviewed by:}

Veljo Kisand, University of Tartu, Estonia

M. Pilar Francino, Center for Public Health Research, Spain

Jer-Horng Wu, National Cheng Kung University, Taiwan

*Correspondence:

Yoshimi Matsumoto, Institute of Scientific and Industrial Research,

Osaka University, Department of Cell Membrane Biology, 8-1 Mihogaoka, Ibaraki, Osaka 567-0047, Japan.

e-mail: yoshimi@sanken.osaka-u.ac.jp

Recently, multidrug-resistant pathogens have disseminated widely owing essentially to their increased multidrug efflux pump activity. Presently, there is a scarcity of new antibacterial agents, and hence, inhibitors of multidrug efflux pumps belonging to the resistancenodulation-cell division (RND) family appear useful in the treatment of infections by multidrug-resistant pathogens. Moreover, recent progress in microfabrication technologies has expanded the application of nano/micro-devices to the field of human healthcare, such as the detection of infections and diagnosis of diseases. We developed a microfluidic channel device for a simple and rapid evaluation of bacterial drug efflux activity. By combining the microfluidic device with a fluorogenic compound, fluorescein-di- $\beta$-Dgalactopyranoside, which is hydrolyzed to a fluorescent dye in the cytoplasm of Escherichia coli, we successfully evaluated the effects of inhibitors on the RND-type multidrug efflux pumps MexAB-OprM and MexXY-OprM from Pseudomonas aeruginosa in E. coli. Our new method successfully detected the MexB-specific inhibitory effect of D13-9001 and revealed an unexpected membrane-permeabilizing effect of Phe-Arg- $\beta$-naphthylamide, which has long been used as an efflux pump inhibitor.

Keywords: fluorescein-di- $\beta$-D-galactopyranoside, microfluidic channel, fluorescence microscopy, pyridopyrimidine, Phe-Arg- $\beta$-naphthylamide, polymyxin B nonapeptide, Escherichia coli, Pseudomonas aeruginosa

\section{INTRODUCTION}

Currently, increase in multidrug resistance among clinical isolates is a major problem in infection control. In particular, the so-called "multidrug-resistant Pseudomonas aeruginosa (MDRP)" which is resistant to major antipseudomonal agents such as carbapenems, quinolones, and aminoglycosides (Sekiguchi et al., 2007b; Kirikae et al., 2008), has been isolated and identified as a cause of nosocomial outbreaks in Japan (Sekiguchi et al., 2007a; Satoh et al., 2008). P. aeruginosa has natural intrinsic resistance tendencies, and MDRP isolates have variable complex resistance mechanisms (Livermore, 2002; Lister et al., 2009). In particular, multidrug efflux pumps, particularly resistance-nodulation-cell division (RND) family pumps, can decrease the sensitivity of $P$. aeruginosa to various compounds (Masuda et al., 2000; Ryan et al., 2001). The RND-type multidrug efflux systems have extremely broad substrate specificities and protect the cells from the actions of antibiotics. They usually function as three-component assemblies spanning the outer and cytoplasmic membranes and the periplasmic space of Gram-negative bacteria. That is, the RND efflux system consists of three different proteins: a cytoplasmic membrane protein (such as MexB), a membrane fusion protein (MexA), and an outer membrane channel (OprM).

Twelve intrinsic efflux systems belonging to the RND family have been identified from the genomic sequence of $P$. aeruginosa (Schweizer, 2003). Among them, MexAB-OprM, MexCD-OprJ, MexEF-OprN, and MexXY efflux systems are known to have important roles in multidrug resistance (Morita et al., 2001; Llanes et al., 2004; Mesaros et al., 2007; Lister et al., 2009). These systems can increase their resistance levels by acquiring additional resistance factors (Henrichfreise et al., 2007; Giske et al., 2008). During the current era of scarcity of new antibacterial agents, RND pump inhibitors in combination with available antibiotics could be useful for treating MDRP infections. Although no clinically useful inhibitor is known presently, the enhancing effects of experimentally available efflux pump inhibitors, namely pyridopyrimidine (D13-9001; Yoshida et al., 2007) and Phe-Arg- $\beta$ naphthylamide (PAßN, MC-207,110; Lomovskaya et al., 2001), on the antibacterial activities of combined antibiotics have been published (Lomovskaya et al., 2001; Mesaros et al., 2007; Tohidpour et al., 2009). Recently, the 3D structures of AcrB (Murakami et al., 2002) and MexB (Sennhauser et al., 2009) and co-crystal structures of AcrB with various substrates were resolved (Murakami et al., 2006; Nakashima et al., 2011), and much information regarding their mechanisms of efflux is now available. At present, rational approaches are being used to develop potent efflux pump inhibitors. However, there is no satisfactory method to directly determine the efflux-inhibiting activities of candidate compounds.

In this review article, we focused on a new technique for a simple and rapid measurement of the activities of bacterial drug efflux pumps and inhibitors by using a microfluidic device recently reported by Matsumoto et al. (2011). We utilized 
an appropriate substrate, fluorescein-di- $\beta$-D-galactopyranoside (FDG), for a visual assay. FDG is non-fluorescent until it is hydrolysed by $\beta$-galactosidase in the cytoplasm of Escherichia coli to produce a highly fluorescent dye, fluorescein (Russo-Marie et al., 1993; Fieldler and Hinz, 1994; Yang and Hu, 2004). We confirmed that both FDG and fluorescein are substrates of RND pumps in $E$. coli. In combination with microfabrication technologies including soft lithography (Whitesides et al., 2001), we constructed a simple microfluidic channel device in order to observe several bacterial cultures simultaneously. By combining FDG and the microfluidic device, we developed a novel and highly sensitive method to evaluate the efflux inhibitory activities of compounds against $P$. aeruginosa MexB and MexY in E. coli and clarified the different action mechanisms of two inhibitors, D13-9001 and PA $\beta \mathrm{N}$.

\section{MATERIALS AND METHODS BACTERIAL STRAINS}

Escherichia coli MG1655 (wild-type), and its efflux pump gene deletion mutants - $\triangle a c r B, \Delta$ tolC, and $\triangle a c r B \Delta$ tolC (Nishino et al., 2008) - were used. The vector plasmid pMMB67HE recombined with efflux pump genes mexABoprM and mexXY-oprM from $P$. aeruginosa (Mokhonov et al., 2004) was transformed with E. coli MG1655 $\triangle a c r B \Delta$ tolC to construct $\triangle a c r B \Delta$ tolC/pMMB67HE $(\Delta \mathrm{BC} / \mathrm{pV}), \Delta$ acrB $\Delta$ tolCl pMMB67HE::mexAB-oprM ( $\triangle \mathrm{BC} / \mathrm{pABM})$, and $\triangle$ acrB $\Delta$ tolCl pMMB67HE::mexXY-oprM ( $\triangle \mathrm{BC} / \mathrm{pXYM})$. For these strains harboring a plasmid, ampicillin (Sigma-Aldrich, Tokyo, Japan) was added to the cultures to ensure retention of the plasmid. Lactose (Sigma-Aldrich) and isopropyl- $\beta$-D-galactopyranoside (IPTG; Sigma-Aldrich) were added to the medium for $\beta$-galactosidase and plasmid-mediated pump inductions, respectively. $P$. aeruginosa IMCJ2.S1 (Sekiguchi et al., 2005) served as the multidrug-resistant strain, and P. aeruginosa PAO1 served as the standard strain.

\section{ANTIBACTERIAL AGENTS AND CHEMICALS}

The antibacterial agents used were aztreonam (Sigma-Aldrich), ciprofloxacin (Tokyo Chemical Industry Co., Ltd., Tokyo, Japan), and erythromycin (Nacalai Tesque, Inc., Kyoto, Japan). D13-9001 (Daiichi Sankyo Co., Tokyo, Japan) and PA $\beta N$ (Sigma-Aldrich) were used as efflux pump inhibitors. Polymyxin B (MERCK KGaA, Darmstadt, Germany) was used as an outer and inner membrane permeabilizer, and Polymyxin B nonapeptide (PMBN; Sigma-Aldrich) was used as an outer membrane permeabilizer.

\section{PREPARATION OF MICROFLUIDIC CHANNELS}

Microfluidic channels (100 $\mu \mathrm{m}$ width, $17 \mu \mathrm{m}$ height, $25-33 \mathrm{~mm}$ length; Figure 1B) fabricated in polydimethylsiloxane (Silpot 184, Dow Corning Toray Co., Ltd., Tokyo, Japan) on a cover glass (Matsunami Glass Ind., Ltd., Osaka, Japan) were prepared by a conventional method described in previous studies (Whitesides et al., 2001; Matsumoto et al., 2011).

\section{EFFLUX PUMP INHIBITION ASSAY}

FDG (Marker Gene Technologies, Inc., Eugene, USA), a fluorogenic compound, is hydrolyzed by $\beta$-galactosidase in the cytoplasm of E. coli to produce a fluorescent dye, fluorescein. Both FDG and fluorescein are substrates of an efflux pump, AcrB, in E. coli.
AcrB effectively prevents FDG influx in wild-type cells, resulting in no fluorescence. The $\Delta a c r B$ and $\triangle$ tolC strains easily imported and hydrolyzed FDG to fluorescein, which is exported by residual pumps in the $\triangle a c r B$ strain. Consequently, fluorescent medium in $\Delta a c r B$ and fluorescent $\Delta$ tol $C$ and $\triangle a c r B \Delta$ tolC cells were observed in the microfluidic channels (Figures 1A,B). The induced activities of $\beta$-galactosidase in strains used in this study were comparable after $a c r B$ and/or tolC deletion and the plasmid-mediated introduction of pumps from $P$. aeruginosa.

The effects of inhibitors on FDG and fluorescein efflux were observed in the microfluidic channel with a BZ-8000 fluorescence microscope (Keyence, Osaka, Japan). Overnight Luria-Bertani (LB) broth (Becton Dickinson and Company, Sparks, USA) cultures were inoculated in fresh LB broth and incubated on a shaker until the culture reached an $\mathrm{OD}_{600}$ of $0.6-0.8$. Lactose $(50 \mathrm{mM})$ was added to induce $\beta$-galactosidase activity in strains harboring no plasmid. LB medium containing $100 \mu \mathrm{g} / \mathrm{ml}$ ampicillin and $1 \mathrm{mM}$ IPTG was used to grow strains harboring the plasmid in order to retain plasmid and induce plasmid-mediated pumps and chromosomal $\beta$-galactosidase. The cultures were premixed with an inhibitor, injected in the microfluidic channels (Figure 1B) with $100 \mu \mathrm{g} / \mathrm{ml} \mathrm{FDG}$, and observed under the microscope after incubation for $15 \mathrm{~min}$ at $37^{\circ} \mathrm{C}$. Different samples containing multiple channels were observed simultaneously in a single image field (Figures 1B-D).

\section{DETERMINATION OF MINIMUM INHIBITORY CONCENTRATION AND CHECKERBOARD MIC}

The minimum inhibitory concentrations (MICs) of the antibacterial agents and checkerboard MICs of these agents in combination with the inhibitors or the membrane permeabilizer were determined using the standard micro dilution method defined by the Clinical and Laboratory Standards Institute (Wayne, PA, USA). Strains harboring the plasmid were cultured in LB medium containing $100 \mu \mathrm{g} / \mathrm{ml}$ ampicillin and $1 \mathrm{mM}$ IPTG. The fractional inhibitory concentration (FIC) indices were calculated as follows: $\mathrm{FIC}=\left[\mathrm{MIC}_{\mathrm{A}}\right.$ in combination $/$ $\mathrm{MIC}_{\mathrm{A} \text { alone }}+\mathrm{MIC}_{\mathrm{B} \text { in combination }} / \mathrm{MIC}_{\mathrm{B} \text { alone }}$. Synergy was defined as an FIC index of less than 0.5 .

\section{RESULTS}

\section{EVALUATION OF INHIBITORS BY THE CLASSIC CHECKERBOARD METHOD}

Checkerboard MIC determination is a simple method to evaluate the effects of inhibitors on antibacterial activity. Although the method can only evaluate the combinatorial effect of an inhibitor on antimicrobials (synergy, addition, indifference, or antagonism), it is possible to estimate the action mechanism of an inhibitor by using different types of antimicrobials and target gene-deleted or gene-introduced mutants. From the viewpoint of efflux, we used three different types of antimicrobial agents in this study. Aztreonam is a substrate of $\mathrm{MexB}$, although ciprofloxacin and erythromycin are substrates of several pumps. Furthermore, from the viewpoint of influx, ciprofloxacin easily penetrates the outer membranes of Gram-negative bacteria, erythromycin hardly penetrates them (Vaara, 1993), while the penetrability of aztreonam appears to be intermediate of the two. Table 1 shows the MICs 
A

\section{FDG Fluorescein AcrB ODOther pumps TolC}

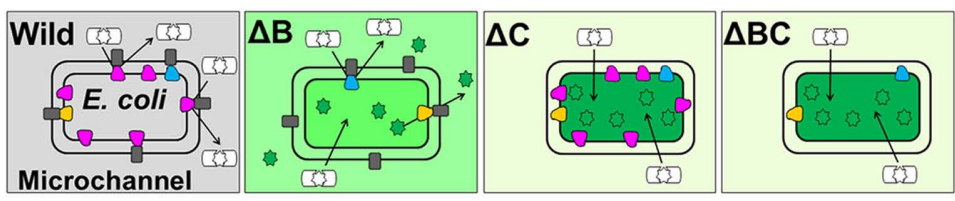

B

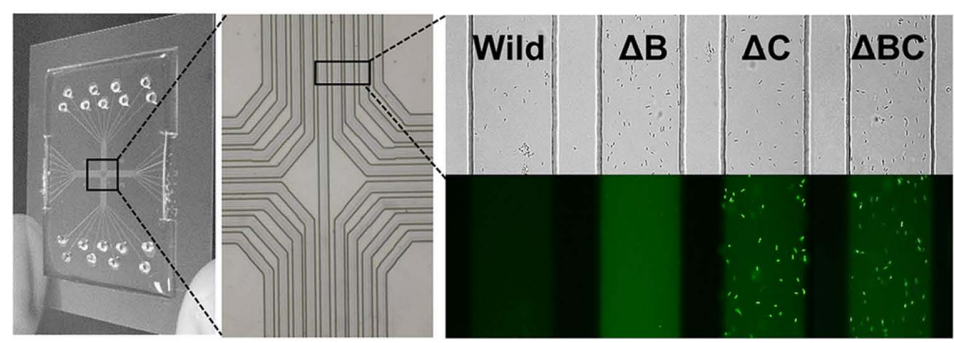

C

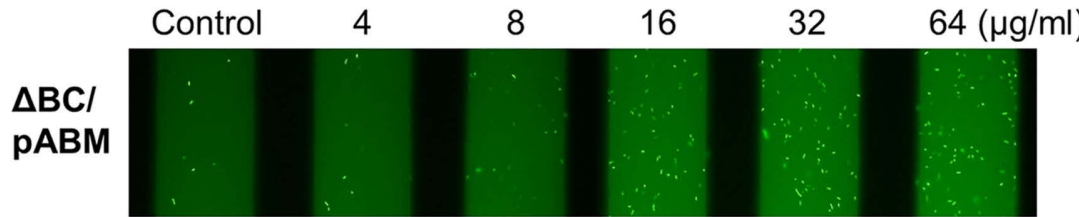

$\triangle B C l$ pXYM

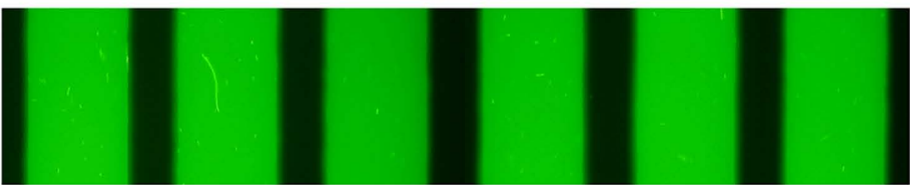

D
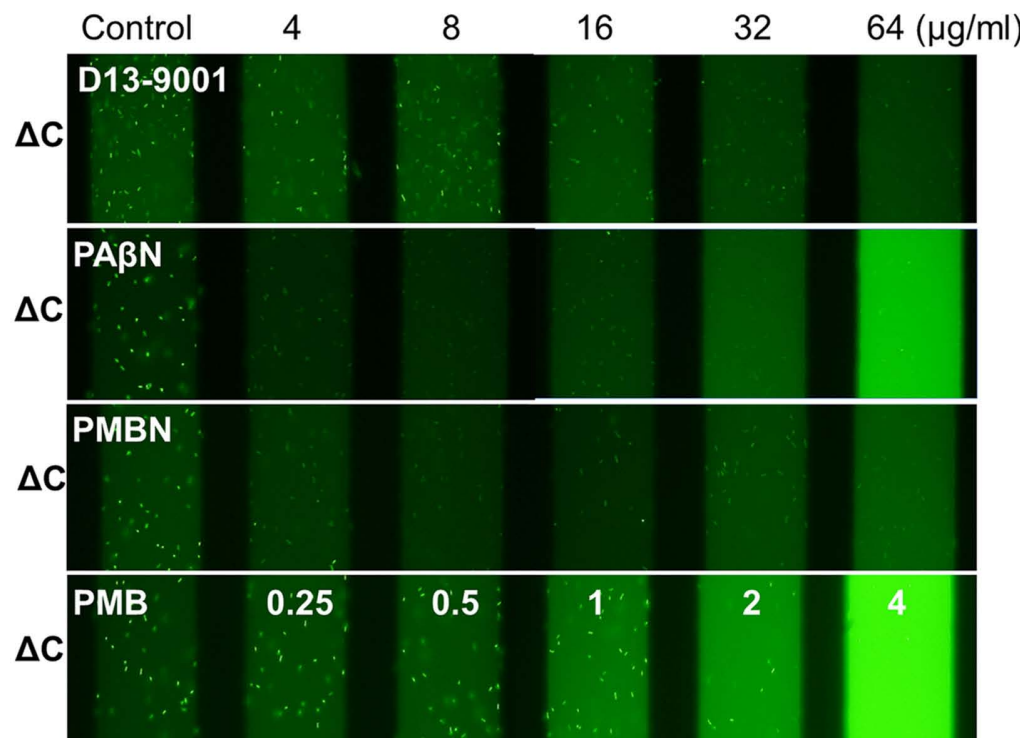

FIGURE 1 |The micro fluidic device and principle of the efflux pump inhibition assay used in this study. (A) Mechanism of the inhibition assay of efflux pumps using FDG as a substrate. (B) Images of the microfluidic device, and an example of the assay: bright-field (top) and fluorescence images (bottom) of the E. coli wild-type, $\Delta \operatorname{acr} B(\Delta \mathrm{B}), \Delta$ tolC $(\Delta \mathrm{C})$, and
$\Delta$ acrB $\Delta$ tolC $(\triangle \mathrm{BC})$ cells. (C) Fuorescence images of the $\Delta$ acrB $\Delta$ tolC/pABM ( $\triangle \mathrm{BC} / \mathrm{pABM}), \Delta$ acrB $\Delta$ tolC/pXYM ( $\triangle \mathrm{BC} / \mathrm{pXYM})$ cells treated with different concentrations of D13-9001. (D) Fluorescence images of the E. coli $\Delta$ tolC cells treated with different concentrations of D13-9001, PA $\beta N$, PMBN, and polymyxin B. Modified from Matsumoto et al. (2011). 
Table 1 | Minimum inhibitory concentrations of agents against tested strains.

\begin{tabular}{|c|c|c|c|c|c|c|c|c|}
\hline Agents & \multicolumn{8}{|c|}{$\mathrm{MIC}(\mu \mathrm{g} / \mathrm{ml})$} \\
\hline ATM & 0.125 & 0.063 & 0.125 & 0.125 & 1 & 0.125 & 2 & 64 \\
\hline CIP & 0.016 & 0.004 & 0.002 & 0.002 & 0.016 & 0.031 & 0.063 & 32 \\
\hline $\mathrm{PA} \beta \mathrm{N}$ & 256 & 64 & 64 & 32 & 256 & 128 & 512 & 512 \\
\hline PMB & 2 & 2 & 2 & 2 & 2 & 2 & 2 & 2 \\
\hline PMBN & $>64$ & 64 & 64 & $>8$ & $>8$ & $>8$ & $>8$ & $>8$ \\
\hline
\end{tabular}

ATM, aztreonam; CIP, ciprofloxacin; ERY, erythromycin; D13-9001, pyridopyrimidine; PABN, Phe-Arg- $\beta$-naphthylamide; PMB, polymyxin B; PMBN, polymyxin B nonapeptide. Table based on the results from Matsumoto et al. (2011).

of agents against strains of $E$. coli and $P$. aeruginosa used in this study. The MIC of aztreonam against $E$. coli was not influenced by $a c r B$ and tolC deletion or plasmid-mediated mexXY-oprM introduction. However, plasmid-mediated mexAB-oprM introduction decreased the sensitivity of $\triangle a c r B \Delta$ tolC to aztreonam by eightfold. By contrast, the MICs of ciprofloxacin and erythromycin against E. coli were decreased by $a c r B$ and/or tolC deletion and increased by plasmid-mediated pseudomonal efflux pump gene (mexAB-oprM or mexXY-oprM) introduction.

There are two famous efflux pump inhibitors: D13-9001 and $\mathrm{PA} \beta \mathrm{N}$. The former is specific for MexB, whereas the latter is nonspecific. $\mathrm{PA} \beta \mathrm{N}$ had weak antibacterial activity against these $E$. coli and $P$. aeruginosa strains, and its MIC was changed by the deletion or introduction of pump genes, which revealed that pump deletion mutants had higher sensitivity to PA $\beta N$ than the wild-type strain. The results indicate that $\mathrm{PA} \beta \mathrm{N}$ is a substrate of RND pumps. D139001 had no antibacterial activity against these strains at concentrations less than $64 \mu \mathrm{g} / \mathrm{ml}$. Inhibitor's concentration-dependent changes in the MICs of antimicrobial agents were compared (Figure 2). Data obtained from $\Delta$ tolC, $\Delta a c r B \Delta$ tolC, and $\Delta \mathrm{BC} / \mathrm{pV}$ were similar for all antimicrobial agents used in this study, and thus, we presented the data for $\triangle \mathrm{BC} / \mathrm{pV}$ as a representative full RND pump deletion mutant in Figure 2.

In E. coli MG1655, D13-9001 acted synergistically with ciprofloxacin and erythromycin against the wild-type strain and had no effect with either agents against $\Delta a c r B, \Delta$ tolC, or $\Delta \mathrm{BC} / \mathrm{pV}$ (Figures 2A,D,G). In $P$. aeruginosa, D13-9001 acted synergistically with aztreonam and ciprofloxacin but not with erythromycin, which had a higher affinity for MexY than for MexB (Table 1), against both sensitive and multidrug-resistant strains, and the synergy between D13-9001 and aztreonam was remarkable in these strains. D13-9001 increased the susceptibilities of E. coli $\triangle \mathrm{BC} / \mathrm{pABM}$ to all three agents and had no effect on the susceptibilities of $\triangle B C / p V$ and $\triangle B C / p X Y M$ (Figures $2 A, D, G$ ).

In contrast, $\mathrm{PA} \beta \mathrm{N}$ significantly increased erythromycin activity against all strains including $\triangle a c r B$ and $\triangle \mathrm{BC} / \mathrm{pV}$ (Figure $2 \mathbf{H}$ ), although the effect of $\mathrm{PA} \beta \mathrm{N}$ on ciprofloxacin activity remained additive against all $E$. coli strains (Figure 2B). However, PA $\beta \mathrm{N}$ exhibited remarkable synergy against MDRP IMCJ2.S1 with all of the three agents (Figures 2B,E,H), and the FIC indices of PA $\beta \mathrm{N}$ were $0.016,0.031$, and 0.063 with ciprofloxacin, aztreonam, and erythromycin, respectively. The effect of PA $\beta \mathrm{N}$ was less strong on PAO1 than on MDRP with ciprofloxacin or aztreonam. Furthermore, $\mathrm{PA} \beta \mathrm{N}$ acted synergistically with aztreonam in $\triangle \mathrm{BC} / \mathrm{pV}$, although this combination was additive in $\triangle \mathrm{BC} / \mathrm{pABM}$ and the wild-type. Synergy between PA $\beta \mathrm{N}$ and these antimicrobial agents could not be explained by efflux pump inhibition by PA $\beta N$.

Other than efflux pump inhibitors, outer membrane permeabilizers have also been used for enhancing the activity of antimicrobial agents (Vaara, 1992; Vaara and Porro, 1996; Vaara et al., 2010; Vingsbo Lundberg et al., 2010). Among them, PMBN is known to permeabilize only the outer membrane of Gram-negative bacteria, whereas polymyxin B permeabilizes both the outer and inner membranes (Vaara and Vaara, 1983a,b; Viljanen and Vaara, 1984). PMBN had also weak antimicrobial activities against pumpdeleted strains, and it appeared to be a substrate of pumps. The effect of PMBN on ciprofloxacin activity was additive against the E. coli strains and PAO1 and was synergistic against IMCJ2.S1. By contrast, $\mathrm{PMBN}$ increased erythromycin activity against all strains including pump deletion mutants $\triangle a c r B, \Delta$ tolC, and $\Delta \mathrm{BC} / \mathrm{pV}$. These results obtained for $\mathrm{PMBN}$ were comparable to and stronger than the results for PA $\beta N$. PMBN also increased aztreonam activity against all strains.

\section{EFFLUX PUMP INHIBITION OBSERVED BY THE NEW DEVICE}

AcrAB-TolC and the analogous RND pumps effectively prevented FDG influx in E. coli wild-type cells, resulting in no fluorescence (Figures 1A,B). By contrast, $\triangle a c r B$ and $\Delta$ tolC easily accumulated and hydrolyzed FDG to fluorescein, which accumulated in $\Delta$ tolC cells but not in $\triangle a c r B$ cells. Consequently, we observed fluorescent medium in $\triangle a c r B$ experiments and fluorescent cells in $\Delta$ tolC experiments. Images of these strains in the microfluidic channels are shown in Figure 1B. Fluorescence was highly accumulated only in tolC-deleted strains. We also evaluated the inhibitors in relation to the MexAB-OprM and MexXY-OprM pumps from $P$. aeruginosa in E. coli. Fluorescent medium was observed in $\triangle \mathrm{BC} / \mathrm{pABM}$ and $\triangle \mathrm{BC} / \mathrm{pXYM}$ (Figure 1C), whereas fluorescent cells were observed in $\triangle \mathrm{BC} / \mathrm{pV}$ like in $\Delta$ tolC (Figure 1D). Thus, 

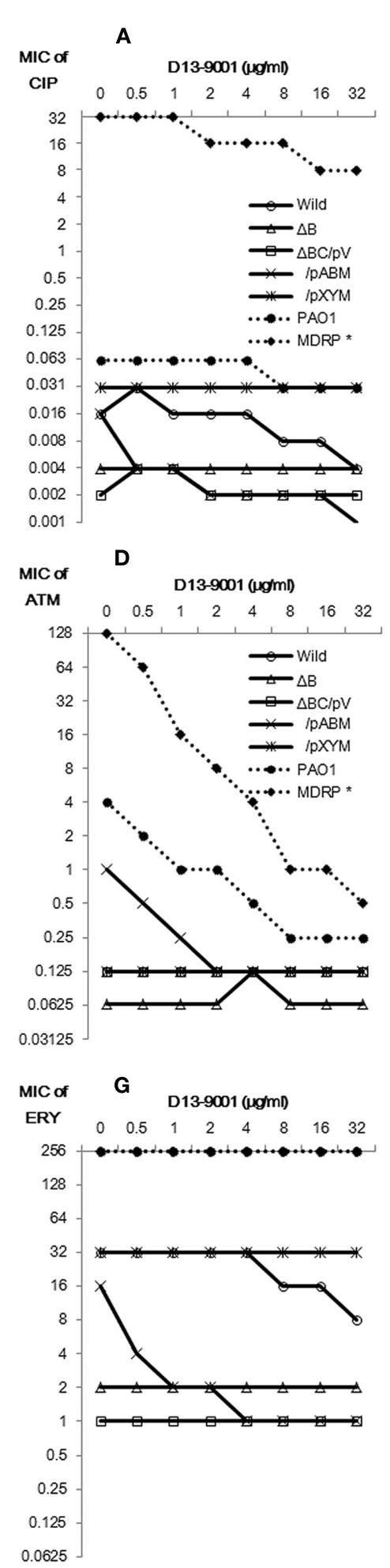
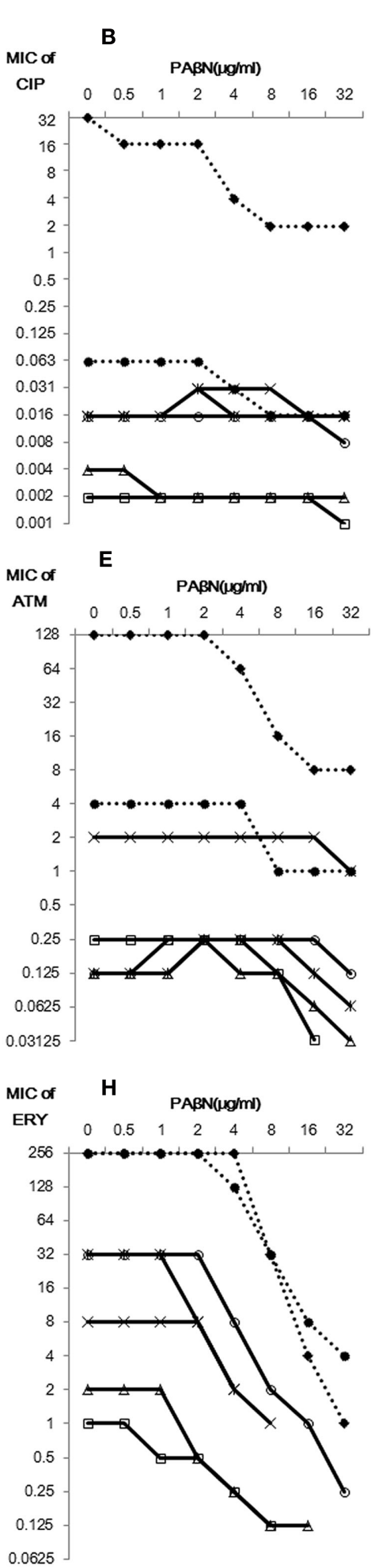

C

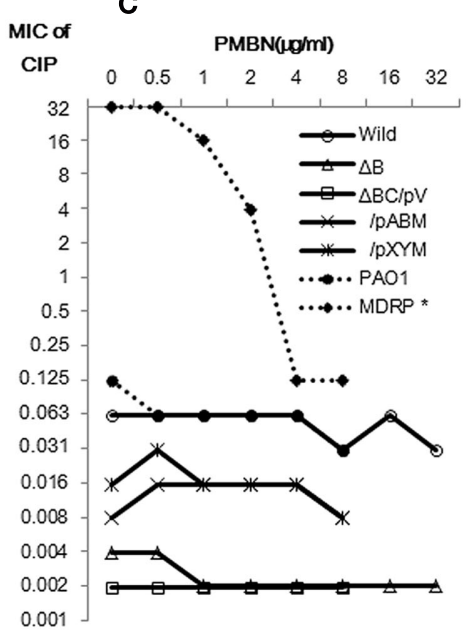

$\mathbf{F}$
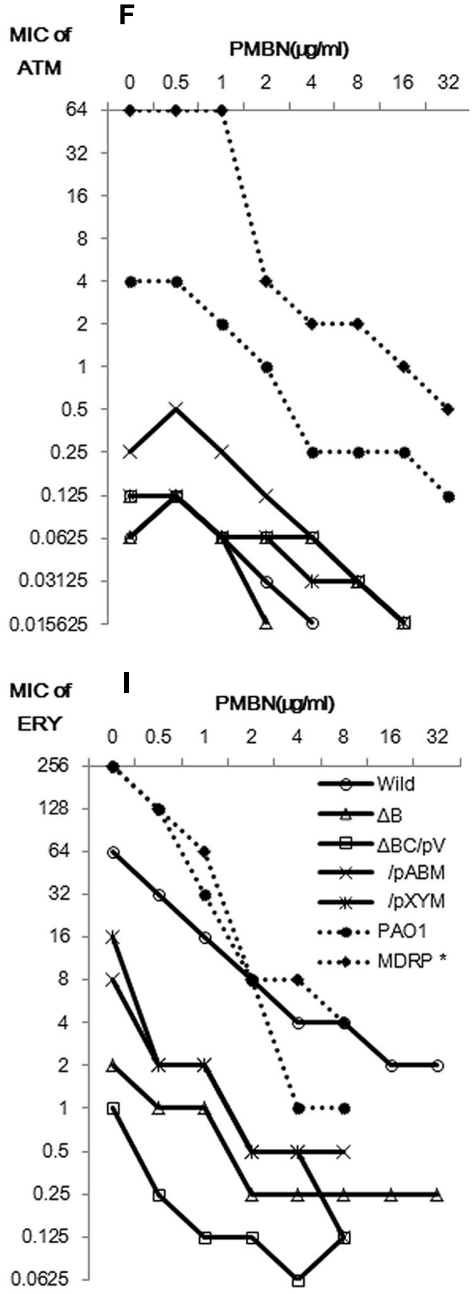

FIGURE 2 | Effect of D13-9001, PA $\beta N$, and PMBN on ciprofloxacin, aztreonam, and erythromycin activity. E. coli MG1655 wild, $\triangle a c r B$, $\triangle$ acrB $\Delta$ tolC/pMMB67HE ( $\triangle \mathrm{BC} / \mathrm{pV}), \Delta$ acrB $\Delta$ tolC/pABM $(\triangle \mathrm{BC} / \mathrm{pABM})$, $\Delta$ acrB $\Delta$ tolC/pXYM ( $\triangle \mathrm{BC} / \mathrm{pXYM})$, P. aeruginosa PAO1, and MDRP
IMCJ2.S1 were used. Changes in the MICs of ciprofloxacin [CIP: (A-C)], aztreonam [ATM: (D-F)], and erythromycin [ERY: (G-I)] induced by D13-9001 (A,D,G), PA $\beta N(\mathbf{B}, \mathbf{E}, \mathbf{H})$, or PMBN (C,F,I) were determined by checkerboard method. Modified from Matsumoto et al. (2011). 
these RND pumps from $P$. aeruginosa appeared to be functional in E. coli $\triangle$ acrB $\Delta$ tolC.

The effect of D13-9001 was not significant in wild-type $E$. coli in the new method (Matsumoto et al., 2011). Fluorescence in the device was decreased to a minor level by D13-9001 in a concentration-dependent manner in $\triangle a c r B$ and $\triangle$ tolC, and the possibility of FDG influx-blocking activity of D13-9001 was suggested. However, D13-9001 clearly increased the accumulation of fluorescein in $\triangle \mathrm{BC} / \mathrm{pABM}$ cells, and the number of fluorescent cells was increased by D13-9001 in a concentration-dependent manner in $\triangle \mathrm{BC} / \mathrm{pABM}$, although it had almost no effect in $\triangle \mathrm{BC} / \mathrm{pXYM}$ (Figure 1C). Conversely, PA $\beta \mathrm{N}$ increased the fluorescence in the medium of all strains, particularly for $\Delta$ tolC (Matsumoto et al., 2011), and the accumulation of fluorescein in the cells of $\Delta$ tolC disappeared (Figure 1D). PA $\beta \mathrm{N}$ appeared to have membrane-permeabilizing activities.

\section{MEMBRANE-PERMEABILIZING ACTIVITY ESTIMATED BY THE NEW DEVICE}

We further evaluated the effect of $\mathrm{PA} \beta \mathrm{N}$ in comparison with those of polymyxin $\mathrm{B}$ and PMBN in $\Delta$ tolC by the device. The membrane-permeabilizing activities of $\mathrm{PA} \beta \mathrm{N}, \mathrm{PMBN}$, and polymyxin Bwere visualized by this new method using the pumpdeficient strain $\Delta$ tolC. The MexB inhibitor D13-9001 had almost no effect on fluorescein distribution in $\triangle$ tolC (Figure 1D). By contrast, fluorescein accumulation in $\Delta$ tolC cells was disappeared by $4 \mu \mathrm{g} / \mathrm{ml} \mathrm{PA} \beta \mathrm{N}$ or by $4 \mu \mathrm{g} / \mathrm{ml} \mathrm{PMBN}$. Polymyxin $\mathrm{B}$ increased the fluorescence in the medium at concentrations exceeding $1 \mu \mathrm{g} / \mathrm{ml}$, but fluorescein accumulation was observed in the presence of $1 \mu \mathrm{g} / \mathrm{ml}$ polymyxin B also. The disappeared accumulation of fluorescein in $\Delta$ tolC cells and increased fluorescence in the medium of $\Delta$ tolC appears to correspond with the outer and inner membrane permeabilization of $E$. coli, respectively. The effect of PA $\beta \mathrm{N}$ was similar to that of PMBN at lower concentrations and was similar to that of polymyxin $\mathrm{B}$ at higher concentrations (Figure 1D). The effect of outer membrane permeabilizers is significant with antibacterial agents that are effectively excluded by the intact outer membrane. The synergistic action of $\mathrm{PA} \beta \mathrm{N}$ in combination with erythromycin appeared to arise from the outer membrane-permeabilizing activity of PA $\beta N$. In E. coli, the outer membrane-permeabilizing activity of PA $\beta \mathrm{N}$ was confirmed by the increased hydrolysis of nitrocefin, and the inner membrane-permeabilizing activity of PA $\beta \mathrm{N}$ was confirmed by the increased accumulation of SYTOX Green (Matsumoto et al., 2011).

\section{DISCUSSION \\ FEATURES OF THE NEW METHOD TO DETERMINE EFFLUX PUMP INHIBITORY ACTIVITIES AND MEMBRANE-PERMEABILIZING ACTIVITIES}

FDG was defined as a substrate of RND pumps because it was more easily hydrolyzed in pump deletion mutants than in wildtype cells. Fluorescein was also defined as a substrate of pumps based on its accumulation in $\triangle$ tolC cells. Furthermore, from the results of the complete blockage of FDG hydrolysis by the addition of a protonophore, carbonyl cyanide $m$-chlorophenylhydrazone (CCCP), in all of the strains including $\Delta$ tolC (data not shown),
FDG was confirmed to be actively imported into the cytoplasm. If CCCP only blocks efflux pumps, increased fluorescence would be observed in wild-type cells. The lactose permease LacY is not an FDG permease because lacY deletion in $\Delta$ lacI mutants (constitutive $\beta$-galactosidase producer) had no effect on FDG hydrolysis in the mutants (data not shown), and we have yet to identify an FDG permease. In wild-type cells, FDG is hardly imported into the cytoplasm because FDG is exported by AcrB from the periplasm before it is trapped by permease. The rate of FDG influx will increase in relation to the concentration of FDG in the periplasm until it reaches its maximum rate. Moderate inhibition of the pumps causes FDG influx and an efflux of fluorescein from the cells by the remaining activity of the pumps, and full inhibition of the pumps results in fluorescein accumulation in cells, similar to what is observed in $\Delta$ tolC cells. A real pump inhibitor without any effect on the bacterial membrane will increase fluorescence in wild-type cells in a concentration-dependent manner and will increase the accumulation of fluorescence in the cells. The microfluidic channel allowed these discriminations to be monitored using fluorescence microscopy. In fact, we detected moderately increasing fluorescence and increased accumulation of fluorescence in $\triangle \mathrm{BC} / \mathrm{pABM}$ in relation to the increasing concentration of D13-9001 (Figure 1C) which is known as a specific inhibiter of the MexAB-OprM pump. D13-9001 had no effect on the MexXY-OprM pump producing E. coli. The combinatorial effects of D13-9001 with the antimicrobials assessed by the checkerboard method are easily understandable by the inhibitory effect of D13-9001 on the AcrB or MexB pump in all of the tested strains of E. coli and P. aeruginosa.

While evaluating the activity of $\mathrm{PA} \beta \mathrm{N}$, we found that our method is also valuable for evaluation of the outer and inner membrane-permeabilizing activities of compounds. Outer membrane permeabilization causes the leakage of fluorescein from $\Delta$ tolC cells, and inner membrane permeabilization efficiently increases FDG influx and fluorescein production, resulting in fluorescein release from cells with or without pumps. By the FDG assay, it is easy to detect outer membrane permeabilization by the disappearance of fluorescein accumulation in $\Delta$ tolC cells and inner membrane permeabilization based on increases in fluorescence, particularly in the medium of pump deletion mutants.

\section{COMPARISON OF OUR NEW METHOD WITH OTHER AVAILABLE METHODS FOR DETERMINING EFFLUX PUMP-INHIBITORY ACTIVITIES}

Several fluorometric methods for evaluating efflux pump inhibitors have been published using substrates of these pumps such as alanine $\beta$-naphthylamide (Lomovskaya et al., 2001), $N$ phenylnaphthylamine (Lomovskaya et al., 2001), ethidium bromide (Lomovskaya et al., 2001), and pyronin Y (Kaatz et al., 2003). However, the inhibitory activities of PA $\beta N$ itself could not be determined by the methods utilizing alanine $\beta$-naphthylamide or $N$-phenylnaphthylamine due to the high background fluorescence (Lomovskaya et al., 2001). Furthermore, PA $\beta N$ has been reported to have no inhibitory effect on the efflux of ethidium bromide (Lomovskaya et al., 2001; Schumacher et al., 2006; Viveiros et al., 2008), which is also a known substrate of ABCtype transporters (Martins et al., 2009). The fluorescence of these compounds was less strong than that of fluorescein, and thus, they 
are not considered suitable for visualization in the microfluidic channel.

The determination of efflux pump inhibition activity via the typical methods of measuring the influx or efflux of some substrates by their fluorescence with a plate reader makes it difficult to exclude the effect of outer membrane-permeabilizing activity. In addition, accurate estimation of FDG hydrolysis by monitoring fluorescence with a plate reader is impractical, because the total fluorescence of fluorescein determined by a plate reader is higher when it diffused in the medium than when it is accumulated into cells; fluorescence determined by a plate reader in this study was generally higher in $\triangle a c r B$ than in $\Delta$ tolC (data not shown). Therefore, fluorescence determined by a plate reader does not accurately correlate with the amount of fluorescein produced, and it is difficult to estimate the inhibitory effect on pumps with FDG by using a plate reader. The microfluidic channel method enables the discrimination of pure efflux pump inhibition from membrane permeabilization. However, when more than two different pumps are present in a cell, it may be difficult to detect the effect of a specific inhibitor on either pump. To overcome this problem, we need a mutant lacking all RND pumps and producing TolC that accumulates fluorescein. Deletions of $a c r B, a c r D, a c r E F, m d t A B C$, and $m d t E F$ were not sufficient to ensure the accumulation of fluorescein in a manner similar to that in $\Delta$ tolC (data not shown). The effect of D13-9001 on E. coli AcrAB-TolC can be detected by this method for a strain that produces only AcrAB-TolC. We would be able to apply this method for evaluation of the efflux pump inhibitors against RND pumps in P. aeruginosa when we construct a strain of $P$. aeruginosa producing both $\beta$-galactosidase and FDG permease. In addition, the microfluidic channels are useful for comparative observation of multiple samples at the same time and easy to tailor-make. We are expanding application of these micro-devices for other microbiological assays.

\section{SUSPICION OF THE EFFLUX PUMP INHIBITORY ACTIVITY OF PA $\beta N$}

Throughout this study, we could not definitively determine whether PA $\beta N$ actually inhibits efflux pumps. Using our new method, we observed that PA $\beta N$ increased FDG hydrolysis and fluorescein leakage in all strains (Matsumoto et al., 2011), particularly in pump deletion mutants (Figure 1D). Because PA $\beta \mathrm{N}$ was reported first, it has been universally recognized as an efflux pump inhibitor (Lomovskaya et al., 2001; Mesaros et al., 2007). The effect of PA $\beta N$ on MDRP IMCJ2.S1 was remarkably synergistic with all the agents examined in the present study (Figure 2). However, PA $\beta \mathrm{N}$ increased the susceptibilities of pump deletion mutants of E. coli especially to erythromycin, although it had almost no effect on ciprofloxacin in E. coli expressing MexABOprM or MexXY-OprM (Figure 2); this suggests that PA $\beta N$ could not inhibit the efflux of ciprofloxacin by MexB or MexY. These actions of PA $\beta \mathrm{N}$ do not correlate with efflux pumps and are similar to those of the outer membrane permeabilizer PMBN. The effect of $\mathrm{PA} \beta \mathrm{N}$ on the outer membrane was already known when it was discovered (Lomovskaya et al., 2001). Previous authors used a nitrocefin hydrolysis assay with intact cells to evaluate the outer membrane-permeabilizing activity of $\mathrm{PA} \beta \mathrm{N}$ in $P$. aeruginosa, and permeabilizing activity was visible at $\mathrm{PA} \beta \mathrm{N}$ concentrations lower than $16 \mu \mathrm{g} / \mathrm{ml}$ on the outer membranes of pump-deficient or
CCCP-applied strains. Nitrocefin is a substrate of efflux pumps (Nagano and Nikaido, 2009), and thus, the permeabilizing activity of agents should be evaluated in the pump-deficient condition. The permeabilizing activity of PA $\beta \mathrm{N}$ on the outer membrane of E. coli by nitrocefin hydrolysis assay was comparable to the activity obtained in the FDG assay (Matsumoto et al., 2011) and was higher than that reported previously for the outer membrane of $P$. aeruginosa (Lomovskaya et al., 2001). The outer membranepermeabilizing activity of $\mathrm{PA} \beta \mathrm{N}$ has also been published by other researchers using resazurin as a substrate (Vidal-Aroca et al., 2009). In the FDG assay, the effect of PA $\beta \mathrm{N}$ on the outer membrane of E. coli was visible at $4 \mu \mathrm{g} / \mathrm{ml}$ (Figure 1D). LB was used for the FDG assay and the results were easy to compare with the results of synergy in MICs with antimicrobial agents in which the effect of $\mathrm{PA} \beta \mathrm{N}$ was also detected in concentrations higher than $4 \mu \mathrm{g} / \mathrm{ml}$. Outer membrane-permeabilizing activity is known to increase the sensitivity of bacteria to antimicrobial agents such as erythromycin that are excluded by an intact outer membrane (Vaara, 2009; Vaara et al., 2010) as well as efflux pump inhibitors, and we could obtain similar results using PMBN. The effect of $\mathrm{PA} \beta \mathrm{N}$ on antimicrobial agents (Figure 2) appears to be due to its activity on the E. coli cell membranes. We could not obtain any clear evidence of the inhibitory activity of PA $\beta \mathrm{N}$ on the efflux pump itself. The possibility that PA $\beta N$ competes with a substrate for binding to the efflux pump cannot be excluded completely, although competitive inhibition of efflux pumps by another substrate was not proven in E. coli by Elkins and Mullis (2007). The precise mechanism of synergy between $\mathrm{PA} \beta \mathrm{N}$ and antimicrobial agents needs to be elucidated in future. Based on our findings using this new method, we concluded that PA $\beta \mathrm{N}$ appears to be a substrate of the pumps and permeabilizes the membranes of E. coli in contrast to D13-9001, which specifically inhibits MexB.

\section{AVAILABILITY OF EFFLUX PUMP INHIBITORS AND MEMBRANE PERMEABILIZERS}

The effect of D13-9001 is simple and specific to strains expressing AcrB or MexB pumps. D13-9001 exhibits maximum synergistic effect with aztreonam, a substrate preferred only by MexB. However, D13-9001 was not completely synergistic with ciprofloxacin, which is a substrate of MexB, MexY, and other pumps. Because various pump structures exist, it may be difficult to develop a super inhibitor that can inhibit all major pumps in P. aeruginosa. Matching an inhibitor with combined antimicrobial agents is essential. Susceptibility augmenting agents such as $\mathrm{PA} \beta \mathrm{N}$ may be useful when used in combination with a substrate of multiple efflux pumps such as erythromycin and ciprofloxacin. Considerably, membrane permeabilizers can enhance activities of antimicrobial agents against efflux pump deficient strains, enabling expansion of the spectrum from targeting anti-Gram positives to anti-Gram negatives. These features can transform old antimicrobial agents to newer and improved ones, and these activities can be evaluated by our new method all together. Discovery of a clinically useful agent augmenting antipseudomonal activities is anticipated.

\section{ACKNOWLEDGMENTS}

This study was supported by the Grant for Promotion of Fundamental Studies in Health Sciences of the National Institute 
of Biomedical Innovation (Project ID 07-03), Grants from the Japan Society for the Promotion of Science, and the Ministry of Education, Culture, Sports, Science, and Technology of Japan. We are grateful to Dr. Taiji Nakae for providing plasmids and to Dr. Kirikae for providing $P$. aeruginosa IMCJ2.S1.

\section{REFERENCES}

Elkins, C. A., and Mullis, L. B. (2007). Substrate competition studies using whole-cell accumulation assays with the major tripartite multidrug efflux pumps of Escherichia coli. Antimicrob. Agents Chemother. 51, 923-929.

Fieldler, F., and Hinz, H. (1994). No intermediate channelling in stepwise hydrolysis of fluorescein di-betaD-galactoside by beta-galactosidase. Eur. J. Biochem. 222, 75-81.

Giske, C. G., Buaro, L., Sundsfjord, A., and Wretlind, B. (2008). Alterations of porin, pumps, and penicillin-binding proteins in carbapenem resistant clinical isolates of Pseudomonas aeruginosa. Microb. Drug Resist. 14, 23-30.

Henrichfreise, B., Wiegand, I., Pfister, W., and Wiedemann, B. (2007). Resistance mechanisms of multiresistant Pseudomonas aeruginosa strains from Germany and correlation with hypermutation. Antimicrob. Agents Chemother. 51, 4062-4070.

Kaatz, G. W., Moudgal, V. V., Seo, S. M., and Kristiansen, J. E. (2003). Phenothiazines and thioxanthenes inhibit multidrug efflux pump activity in Staphylococcus aureus. Antimicrob. Agents Chemother. 47, 719-726.

Kirikae, T., Mizuguchi, Y., and Arakawa, Y. (2008). Investigation of isolation rates of Pseudomonas aeruginosa with and without multidrug resistance in medical facilities and clinical laboratories in Japan. J. Antimicrob. Chemother. 61, 612-615.

Lister, P. D., Wolter, D. J., and Hanson, N. D. (2009). Antibacterialresistant Pseudomonas aeruginosa: clinical impact and complex regulation of chromosomally encoded resistance mechanisms. Clin. Microbiol. Rev. 22, 582-610.

Livermore, D. M. (2002). Multiple mechanisms of antimicrobial resistance in Pseudomonas aeruginosa: our worst nightmare? Clin. Infect. Dis. 34, 634-640.

Llanes, C., Hocquet, D., Vogne, C., Benali-Baitich, D., Neuwirth, C., and Plesiat, P. (2004). Clinical strains of Pseudomonas aeruginosa overproducing MexAB-OprM and MexXY efflux pumps simultaneously. Antimicrob. Agents Chemother. 48, 1797-1802.
Lomovskaya, O., Warren, M. S., Lee, A., Galazzo, J., Fronko, R., Lee, M., Blais, J., Cho, D., Chamberland, S., Renau, T., Leger, R., Hecker, S., Watkins, W., Hoshino, K., Ishida, H., and Lee, V. J. (2001). Identification and characterization of inhibitors of multidrug resistance efflux pumps in Pseudomonas aeruginosa: novel agents for combination therapy. Antimicrob. Agents Chemother. 45, 105-116.

Martins, A., Spengler, G., Rodrigues, L., Viveiros, M., Ramos, J., Martins, M., Couto, I., Fanning, S., Pages, J. M., Bolla, J. M., Molnar, J., and Amaral, L. (2009). pH Modulation of efflux pump activity of multidrug resistant Escherichia coli: protection during its passage and eventual colonization of the colon. PLoS ONE 4, e6656. doi:10.1371/journal.pone.0006656

Masuda, N., Sakagawa, E., Ohya, S., Gotoh, N., Tsujimoto, H., and Nishino, T. (2000). Substrate specificities of MexAB-OprM, MexCDOprJ, and MexXY-oprM efflux pumps in Pseudomonas aeruginosa. Antimicrob. Agents Chemother. 44, 3322-3327.

Matsumoto, Y., Hayama, K., Sakakihara, S., Nishino, K., Noji, H., Iino, R., and Yamaguchi, A. (2011). Evaluation of multidrug efflux pump inhibitors by a new method using microfluidic channels. PLoS ONE 6, e18547. doi:10.1371/journal.pone. 0018547

Mesaros, N., Glupczynski, Y., Avrain, L. Caceres, N. E., Tulkens, P. M., and Van Bambeke, F. (2007). A combined phenotypic and genotypic method for the detection of Mex efflux pumps in Pseudomonas aeruginosa. J. Antimicrob. Chemother. 59, 378-386.

Mokhonov, V. V., Mokhonova, E. I., Akama, H., and Nakae, T. (2004). Role of the membrane fusion protein in the assembly of resistancenodulation-cell division multidrug efflux pump in Pseudomonas aeruginosa. Biochem. Biophys. Res. Commun. 322, 483-489.

Morita, Y., Kimura, N., Mima, T., Mizushima, T., and Tsuchiya, T. (2001). Roles of MexXY- and MexAB-multidrug efflux pumps in intrinsic multidrug resistance of Pseudomonas aeruginosa PAO1. J. Gen. Appl. Microbiol. 47, 27-32.
Murakami, S., Nakashima, R., Yamashita, E., Matsumoto, T., and Yamaguchi, A. (2006). Crystal structures of a multidrug transporter reveal a functionally rotating mechanism. Nature 443, 173-179.

Murakami, S., Nakashima, R., Yamashita, E., and Yamaguchi, A. (2002). Crystal structure of bacterial multidrug efflux transporter AcrB. Nature 419, 587-593.

Nagano, K., and Nikaido, H. (2009). Kinetic behavior of the major multidrug efflux pump AcrB of Escherichia coli. Proc. Natl. Acad. Sci. U.S.A. 106, 5854-5858.

Nakashima, R., Sakurai, K., Yamasaki, S., Nishino, K., and Yamaguchi, A. (2011). Structures of the multidrug exporter AcrB reveal a proximal multisite drug-binding pocket. Nature 480, 565-569.

Nishino, K., Senda, Y., and Yamaguchi, A. (2008). The AraC-family regulator GadX enhances multidrug resistance in Escherichia coli by activating expression of mdtEF multidrug efflux genes. J. Infect. Chemother. 14, 23-29.

Russo-Marie, F., Roederer, M., Sager, B., Herzenberg, L. A., and Kaiser, D. (1993). Beta-galactosidase activity in single differentiating bacterial cells. Proc. Natl. Acad. Sci. U.S.A. 90 8194-8198.

Ryan, B. M., Dougherty, T. J., Beaulieu, D., Chuang, J., Dougherty, B. A., and Barrett, J. F. (2001). Efflux in bacteria: what do we really know about it? Expert Opin. Investig. Drugs 10, 1409-1422.

Satoh, R., Tsukada, H., Tanabe, Y. Tamura, Y., Yamamoto, T., Takano, M., Ozaki, K., Tamura, T., and Gejyo, F. (2008). An outbreak and isolation of drug-resistant Pseudomonas aeruginosa at Niigata University Hospital, Japan. J. Infect. Chemother. 14, 325-329.

Schumacher, A., Steinke, P., Bohnert, J. A., Akova, M., Jonas, D., and Kern, W. V. (2006). Effect of 1-(1-naphthylmethyl)-piperazine, a novel putative efflux pump inhibitor, on antimicrobial drug susceptibility in clinical isolates of Enterobacteriaceae other than Escherichia coli. J. Antimicrob. Chemother. 57, 344-348.

Schweizer, H. P. (2003). Efflux as a mechanism of resistance to antimicrobials in Pseudomonas aeruginosa and related bacteria: unanswered questions. Genet. Mol. Res. 2, 48-62.

Sekiguchi, J., Asagi, T., MiyoshiAkiyama, T., Fujino, T., Kobayashi, I., Morita, K., Kikuchi, Y., Kuratsuji, T., and Kirikae, T. (2005). Multidrug-resistant Pseudomonas aeruginosa strain that caused an outbreak in a neurosurgery ward and its aac $\left(6^{\prime}\right)$-Iae gene cassette encoding a novel aminoglycoside acetyltransferase. Antimicrob. Agents Chemother. 49, 3734-3742.

Sekiguchi, J., Asagi, T., MiyoshiAkiyama, T., Kasai, A., Mizuguchi, Y., Araake, M., Fujino, T., Kikuchi, H., Sasaki, S., Watari, H., Kojima, T., Miki, H., Kanemitsu, K., Kunishima, H., Kikuchi, Y., Kaku, M., Yoshikura, H., Kuratsuji, T., and Kirikae, T. (2007a). Outbreaks of multidrug-resistant Pseudomonas aeruginosa in community hospitals in Japan. J. Clin. Microbiol. 45, 979-989.

Sekiguchi, J., Teruya, K., Horii, K., Kuroda, E., Konosaki, H. Mizuguchi, Y., Araake, M., Kawana, A., Yoshikura, H., Kuratsuji, T., Miyazaki, H., and Kirikae, T. (2007b). Molecular epidemiology of outbreaks and containment of drug-resistant Pseudomonas aeruginosa in a Tokyo hospital. J. Infect. Chemother. 13, 418-422.

Sennhauser, G., Bukowska, M. A., Briand, C., and Grutter, M. G. (2009). Crystal structure of the multidrug exporter MexB from Pseudomonas aeruginosa. J. Mol. Biol. 389, 134-145.

Tohidpour, A., Peerayeh, S. N., Mehrabadi, J. F., and Yazdi, H. R. (2009). Determination of the efflux pump-mediated resistance prevalence in Pseudomonas aeruginosa, using an efflux pump inhibitor. Curr. Microbiol. 59, 352-355.

Vaara, M. (1992). Agents that increase the permeability of the outer membrane. Microbiol. Rev. 56, 395-411.

Vaara, M. (1993). Outer membrane permeability barrier to azithromycin, clarithromycin, and roxithromycin in gram-negative enteric bacteria. Antimicrob. Agents Chemother. 37, 354-356.

Vaara, M. (2009). New approaches in peptide antibiotics. Curr. Opin. Pharmacol. 9, 571-576. 
Vaara, M., and Porro, M. (1996). Group of peptides that act synergistically with hydrophobic antibiotics against gram-negative enteric bacteria. Antimicrob. Agents Chemother. 40, 1801-1805.

Vaara, M., Siikanen, O., Apajalahti, J., Fox, J., Frimodt-Moller, N., He, H., Poudyal, A., Li, J., Nation, R. L., and Vaara, T. (2010). A novel polymyxin derivative that lacks the fatty acid tail and carries only three positive charges has strong synergism with agents excluded by the intact outer membrane. Antimicrob. Agents Chemother. 54, 3341-3346.

Vaara, M., and Vaara, T. (1983a). Polycations as outer membranedisorganizing agents. Antimicrob. Agents Chemother. 24, 114-122.

Vaara, M., and Vaara, T. (1983b). Polycations sensitize enteric bacteria to antibiotics. Antimicrob. Agents Chemother. 24, 107-113.

Vidal-Aroca, F., Meng, A., Minz, T., Page, M. G. P., and Dreier, J. (2009).
Use of resazurin to detect mefloquine as an efflux-pump inhibitor in Pseudomonas aeruginosa and Escherichia coli. J. Microbiol. Methods 79, 232-237.

Viljanen, P., and Vaara, M. (1984). Susceptibility of gram-negative bacteria to polymyxin $\mathrm{B}$ nonapeptide. Antimicrob. Agents Chemother. 25, 701-705.

Vingsbo Lundberg, C., Vaara, T., Frimodt-Moller, N., and Vaara, M. (2010). Novel polymyxin derivatives are effective in treating experimental Escherichia coli peritoneal infection in mice. J. Antimicrob. Chemother. 65, 981-985.

Viveiros, M., Martins, A., Paixao, L., Rodrigues, L., Martins, M., Couto, I., Fahnrich, E., Kern, W. V., and Amaral, L. (2008). Demonstration of intrinsic efflux activity of Escherichia coli K-12 AG100 by an automated ethidium bromide method. Int. J. Antimicrob. Agents 31, 458-462.

Whitesides, G. M., Ostuni, E., Takayama, S., Jiang, X. Y., and Ingber, D. E.
(2001). Soft lithography in biology and biochemistry. Annu. Rev. Biomed. Eng. 3, 335-373.

Yang, N. C., and Hu, M. L. (2004). A fluorimetric method using fluorescein di-beta-D-galactopyranoside for quantifying the senescenceassociated beta-galactosidase activity in human foreskin fibroblast Hs68 cells. Anal. Biochem. 325, 337-343.

Yoshida, K., Nakayama, K., Ohtsuka, M. Kuru, N., Yokomizo, Y., Sakamoto, A., Takemura, M., Hoshino, K., Kanda, H., Nitanai, H., Namba, K., Imamura, Y., Zhang, J. Z., Lee, V. J., and Watkins, W. J. (2007). MexAB-OprM specific efflux pump inhibitors in Pseudomonas aeruginosa. Part 7: highly soluble and in vivo active quaternary ammonium analogue D13-9001, a potential preclinical candidate. Bioorg. Med. Chem. 15, 7087-7097.

Conflict of Interest Statement: The authors declare that the research was conducted in the absence of any commercial or financial relationships that could be construed as a potential conflict of interest.

Received: 31 October 2011; accepted: 26 January 2012; published online: 08 February 2012.

Citation: Iino R, Nishino K, Noji H, Yamaguchi $A$ and Matsumoto $Y$ (2012) A microfluidic device for simple and rapid evaluation of multidrug efflux pump inhibitors. Front. Microbio. 3:40. doi: 10.3389/fmicb.2012.00040

This article was submitted to Frontiers in Antimicrobials, Resistance and Chemotherapy, a specialty of Frontiers in Microbiology.

Copyright (C) 2012 Iino, Nishino, Noji, Yamaguchi and Matsumoto. This is an open-access article distributed under the terms of the Creative Commons Attribution Non Commercial License, which permits non-commercial use, distribution, and reproduction in other forums, provided the original authors and source are credited. 